\title{
Application of machine learning tools: Potential and useful approach for the prediction of type 2 diabetes mellitus based on the gut microbiome profile
}

\author{
XIAOCHUN GE ${ }^{1}$, AIMIN ZHANG ${ }^{1}$, LIHUI LI ${ }^{1}$, QITIAN SUN ${ }^{1}$, JIANQIU HE ${ }^{1}$, YU WU $^{2,3}$, RUNDONG TAN $^{2,3}$, \\ YINGXIA PAN $^{2,3}$, JIANGMAN ZHAO ${ }^{2,3}$, YUE XU $^{2,3}$, HUI TANG $^{2,3}$ and YU GAO $^{1}$ \\ ${ }^{1}$ Department of Endocrinology, Affiliated Hospital of Chengde Medical University, \\ Chengde, Hebei 067000; ${ }^{2}$ Shanghai Biotecan Pharmaceuticals Co., Ltd.; \\ ${ }^{3}$ Shanghai Zhangjiang Institute of Medical Innovation, Shanghai 201204, P.R. China
}

Received October 11, 2021; Accepted February 9, 2022

DOI: $10.3892 / \mathrm{etm} .2022 .11234$

\begin{abstract}
The gut microbiota plays an important role in the regulation of the immune system and the metabolism of the host. The aim of the present study was to characterize the gut microbiota of patients with type 2 diabetes mellitus (T2DM). A total of 118 participants with newly diagnosed T2DM and 89 control subjects were recruited in the present study; six clinical parameters were collected and the quantity of 10 different types of bacteria was assessed in the fecal samples using quantitative PCR. Taking into consideration the six clinical variables and the quantity of the 10 different bacteria, 3 predictive models were established in the training set and test set, and evaluated using a confusion matrix, area under the receiver operating characteristic curve (AUC) values, sensitivity (recall), specificity, accuracy, positive predictive value and negative predictive value (npv). The abundance of Bacteroides, Eubacterium rectale and Roseburia inulinivorans was significantly lower in the T2DM group compared with the control group. However, the abundance of Enterococcus was significantly higher in the T2DM group compared with the control group. In addition, Faecalibacterium prausnitzii, Enterococcus and Roseburia inulinivorans were significantly associated with sex status while Bacteroides, Bifidobacterium, Enterococcus and Roseburia inulinivorans were significantly associated with older age. In the training set, among the three models, support vector machine (SVM) and
\end{abstract}

Correspondence to: Ms. Hui Tang, Shanghai Biotecan Pharmaceuticals Co., Ltd., 180 Zhangheng Road, Shanghai 201204, P.R. China

E-mail: tang11_23@126.com

Dr Yu Gao, Department of Endocrinology, Affiliated Hospital of Chengde Medical University, 36 Nanyingzi Street, Chengde, Hebei 067000, P.R. China

E-mail: yugao815@163.com

Key words: type 2 diabetes mellitus, machine learning tools, gut microbiome, Bifidobacterium, Roseburiainulinivorans
XGboost models obtained AUC values of 0.72 and 0.70 , respectively. In the test set, only SVM obtained an AUC value of 0.77 , and the precision and specificity were both above 0.77 , whereas the accuracy, recall and npv were above 0.60. Furthermore, Bifidobacterium, age and Roseburia inulinivorans played pivotal roles in the model. In conclusion, the SVM model exhibited the highest overall predictive power, thus the combined use of machine learning tools with gut microbiome profiling may be a promising approach for improving early prediction of T2DM in the near feature.

\section{Introduction}

Type 2 diabetes mellitus (T2DM) is one of the most common metabolic disorders worldwide and is primarily caused by defective insulin secretion (1). Over the past 30 years, the number of individuals with T2DM and prediabetes has increased two-fold globally, indicating T2DM as a rapidly growing public health challenge. However, T2DM is a multifactorial disease that slowly progresses over several years (2). Environmental factors including obesity, aging, an unhealthy diet, a lack of physical activity, smoking, as well as genetic factors and epigenetic modifications all contribute to the accelerating diabetes epidemic in China (3). However, genetic variation accounts for only a small ratio of risk of T2DM development and environmental factors play a pivotal role in driving the progression of T2DM. In addition, several studies have reported increased hypertension rates among T2DM subjects (4-6). It is estimated that the incidence of hypertension is increased $\sim$ two-fold in patients with T2DM compared with those without T2DM (7). A previous study indicated that moderate consumption of alcohol has been associated with a reduced risk of T2DM (8). However, moderate drinking needs to be monitored cautiously under a culturally appropriate context, particularly considering the stable increase in alcohol consumption in several Asian countries (9) and the excess increase in alcohol consumption in European countries (10).

Recent studies reported the occurrence of gut microbiota (GM) dysbiosis in obese patients with T2DM and indicated that the gut microflora may be a major environmental factor 
involved in the onset and progression of diabetes. Additionally, intestinal microbiome changes were also associated with the onset of type $1 \mathrm{DM}$ and gestational DM $(11,12)$. Therefore, it is necessary to develop a reliable early method for detecting T2DM that could lead to earlier interventions and treatments for T2DM.

The human gut microbiome has been demonstrated to possess 500-1,000 bacterial species, which are estimated to encompass $\sim 2,000,000$ genes. Surprisingly, the bacterial genes possess 100 times more genes than the human genes (13). The GM is a very diversified ecosystem and its function is dependent on several factors, such as host genetics, species, sex, age, body mass index (BMI), diet, smoking and drugs $(14,15)$. GM may be key to the management of T2DM development. The aim of the present study was to develop a rapid machine learning-based method to predict the risk of T2DM.

\section{Materials and methods}

Sample and clinical data collection. A total of 118 newly diagnosed patients with T2DM and 89 controls (non-T2DM) were randomly recruited between January 2019 and October 2020 from the affiliated Hospital of Chengde Medical University (Chengde, China). The inclusion criteria for T2DM were as follows: i) Patients with T2DM were enrolled in accordance with the 1999 WHO diagnostic criteria as previously described (16); and ii) were aged $>18$ years. The exclusion criteria were as follows: i) Acute infection, trauma or surgery within the past month; ii) use of antibiotics, glucocorticoids or other immune regulators within the past month; iii) severe coronary heart disease, stroke or malignant disease; iv) pregnancy or lactation; v) autoimmune diseases, such as hyperthyroidism; and vi) other types of diabetes (17). The exclusion criteria for the controls were the same as those aforementioned.

All procedures were performed and approved (approval no. CYFYLL2021171) in accordance with the ethical standards of the Clinical Research Ethics Committee of the affiliated Hospital of Chengde Medical University (Chengde, China), and written informed consent was obtained from all participants included in the study.

Fecal sample collection and DNA extraction. A total of 207 fresh fecal samples were collected in sterile collection tubes (Thermo Fisher Scientific, Inc.). All samples were stored at $-20^{\circ} \mathrm{C}$ for temporary preservation and then transferred to $-80^{\circ} \mathrm{C}$ for longer term storage. Specifically, $200 \mathrm{mg}$ fecal sample was added to $1 \mathrm{ml}$ PBS in a $1.5 \mathrm{ml}$ tube, vortexed at maximum speed for $3 \mathrm{~min}$ and centrifuged at $167.7 \mathrm{x} \mathrm{g}$ for $5 \mathrm{~min}$ at $4^{\circ} \mathrm{C}$ and then the supernatant was collected and transferred to a 2-ml tube. A total of $\sim 800 \mu 1$ supernatant was centrifuged at $1,677 \mathrm{x} \mathrm{g}$ for $5 \mathrm{~min}$ at $4^{\circ} \mathrm{C}$ and then the supernatant was removed. Subsequently, the microbial DNA was extracted from the fecal samples using a nucleic acid extraction kit (cat. no. T221S) according to the manufacturer's protocol (Xi'an Tianlong Science \& Technology Co., Ltd,). Finally, $\sim 60 \mu$ l DNA was obtained for downstream experiments.

Primers and PCR amplification. A total of 10 microbial oligonucleotide primers were synthesized and purified by General Biol (generalbiol.com/) (Table SI). Quantitative PCR (qPCR) was performed using an ABI-7500 real-time PCR system (Thermo Fisher Scientific, Inc.). The thermocycling conditions were: Pre-denaturation at $95^{\circ} \mathrm{C}$ for $10 \mathrm{~min}$; followed by 45 cycles of denaturation at $95^{\circ} \mathrm{C}$ for $15 \mathrm{sec}$ and annealing at $60^{\circ} \mathrm{C}$ for $45 \mathrm{sec}$. Following amplification, melting temperature analysis of PCR products was performed to determine the specificity of the PCR amplification. The melting curves were obtained by heating from 60 to $95^{\circ} \mathrm{C}$ at a rate of $0.3^{\circ} \mathrm{C} / \mathrm{sec}$, with continuous fluorescence measurement. Differences in threshold cycles between the positive control (universal 16S rDNA) and each bacteria were quantified using the $2^{-\mathrm{ACq}}$ method as previously described (18), where $\Delta \mathrm{Cq}$ was the differences in $\mathrm{Cq}$ values for each bacteria and universal 16S rDNA and the relative abundance of each bacteria was calculated.

Construction of the prediction models. In the present study, three machine learning tools were established to predict T2DM development, including an artificial neural network of the multilayer perceptron (MLP) model, an XGboost model and a support vector machine (SVM) model and combined 6 clinical features and 10 bacterial species. The SVM model has been reported to predict chronic kidney disease in clinical applications (19), the XGBoost model exhibits improved performance in predicting patients with postoperative sepsis (20), and the MLP model performed well when applied to computed tomography for coronary artery disease and myocardial perfusion (21). K-fold is a common cross validation approach, particularly when the datasets are limited (22). Therefore, $\mathrm{k}$-fold $(\mathrm{k}=5)$ was used to train, construct and compare the three predictive models. Additionally, the parameters of the three predictive models were tuned for the optimization of the equations in Python (Table SII).

A total of 207 participants (118 patients with T2DM and 89 controls) were randomly allocated into a training set $(80 \%)$ and a test set $(20 \%)$. In the training set, $\mathrm{k}=5$ was used and various parameter combinations were exhausted using grid search. For each model, the confusion matrix, area under the receiver operating characteristic (ROC) curve (AUC), accuracy, sensitivity (recall), specificity, positive predictive value [ppv (precision)] and negative predictive value (npv) and were used to evaluate and compare the comprehensive performance of feature selection as previously described (23).

Statistical analysis. The three models were used to predict the risk of T2DM and evaluated using Python (version 3.6.12; Python Software Foundation) and incorporated including 6 clinical features and 10 bacterial species. The diagnostic values of the three models were assessed using ROC analysis. After preprocessing the data with pandas and sklearn, XGboost was used to analyze the importance of features and evaluated by Python as previously described (24). Categorical variables were presented by numbers or proportions, and differences in distribution between the two groups were analyzed using a $\chi^{2}$ test in SPSS (version 19.0; IBM Corp.) Continuous variables are presented as the median and range. Continuous variables between the two groups were compared using a nonparametric Mann-Whitney U test (for two groups) or nonparametric Kruskal-Wallis test followed by Dunn's post hoc test (for more than two groups). Statistical calculations were performed in GraphPad Prism (version 8.0; GraphPad Software, Inc.). 


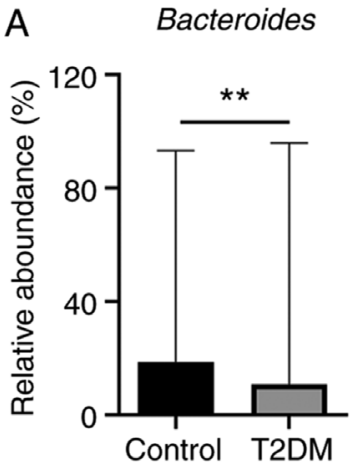

C Roseburia inulinivorans

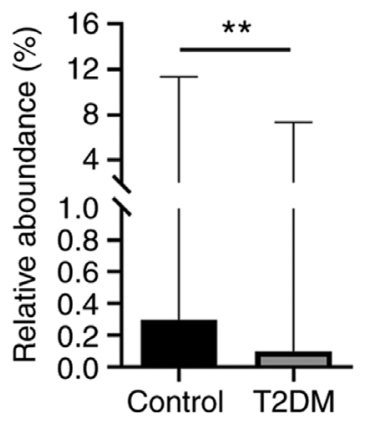

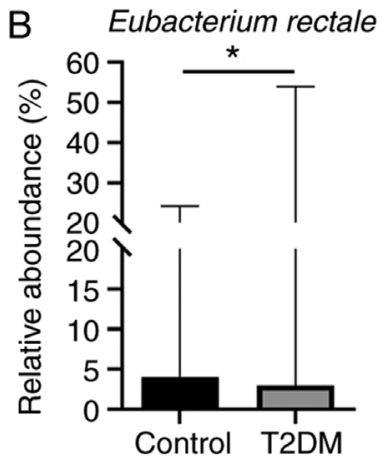

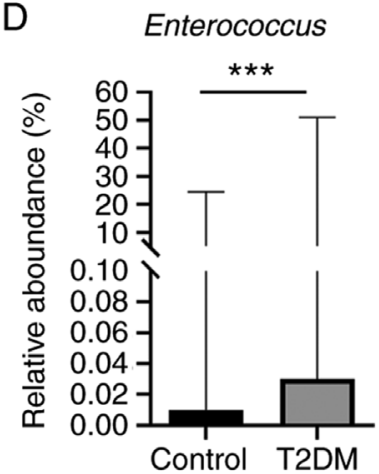

Figure 1. Comparison of 4 bacteria between the patients with T2DM and controls. Abundance of (A) Bacteroides, (B) Eubacterium rectale and (C) Roseburia inulinivorans were significantly lower in the T2DM group than in the control group. (D) The abundance of Enterococcus was significantly higher in the T2DM group than in the control group. Results represent the median and range. ${ }^{*} \mathrm{P}<0.05,{ }^{* * *} \mathrm{P}<0.01$ and ${ }^{* * *} \mathrm{P}<0.001$ as determined by nonparametric Mann-Whitney $\mathrm{U}$ test. T2DM, type 2 diabetes mellitus.

$\mathrm{P} \leq 0.05$ was considered to indicate a statistically significant difference.

\section{Results}

Clinical characteristics of the participants. The clinical characteristics of the patients with T2DM and controls are shown in Table SIII. There were no significant differences in age $(\mathrm{P}=0.502)$, sex $(\mathrm{P}=0.683)$, $\mathrm{BMI}(\mathrm{P}=0.230)$, smoking $(\mathrm{P}=0.146)$, alcohol consumption $(\mathrm{P}=0.220)$ and hypertension status $(\mathrm{P}=0.055)$ between patients with $\mathrm{T} 2 \mathrm{DM}$ and controls.

Comparison of the 10 bacteria between patients with T2DM and controls. A total of 10 bacteria, including Veillonellaceae, Clostridium leptum, Roseburia inulinivorans, Bacteroides, Prevotella, Bifidobacterium, Lactobacillus, Faecalibacterium prausnitzii, Enterococcus and Eubacterium rectale were detected by qPCR. The abundance of Bacteroides $(\mathrm{P}=0.0055)$, Eubacterium rectale $(\mathrm{P}=0.0432)$ and Roseburia inulinivorans $(\mathrm{P}=0.0019)$ was significantly lower in the T2DM group than in the control group (Fig. 1A-C). In addition, the abundance of Enterococcus $(\mathrm{P}=0.0002)$ was significantly higher in the T2DM group than in the control group (Fig. 1D). However, there were no significant differences in Prevotella $(\mathrm{P}=0.164)$, Bifidobacterium $(\mathrm{P}=0.103)$, Veillonellaceae $(\mathrm{P}=0.642)$, Faecalibacterium prausnitzii $(\mathrm{P}=0.157)$, Lactobacillus $(\mathrm{P}=0.078)$ and Clostridium leptum $(\mathrm{P}=0.493)$ between the two groups (Fig. S1).

In addition, Faecalibacterium prausnitzii was significantly higher in the control female subgroup than in the T2DM female subgroup ( $\mathrm{P}=0.032$; Fig. 2A). Furthermore, the abundance of Enterococcus was higher in the T2DM male subgroup than in both control female $(\mathrm{P}=0.025)$ and male $(\mathrm{P}=0.0121)$ subgroups (Fig. 2B). Roseburia inulinivorans was significantly higher in both control female and male subgroups than in the T2DM female subgroup $(\mathrm{P}=0.0008$ and $\mathrm{P}=0.0026$, respectively) (Fig. 2C). However, there were no significant differences in the abundance of the Bacteroides $(\mathrm{P}=0.0477)$, Prevotella $(\mathrm{P}=0.468)$, Bifidobacterium $(\mathrm{P}=0.35)$, Lactobacillus $(\mathrm{P}=0.326)$, Eubacterium rectale $(\mathrm{P}=0.118)$, Veillonellaceae $(\mathrm{P}=0.124)$ and Clostridium leptum $(\mathrm{P}=0.178)$ between each subgroup (Fig. S2).

The abundance of Bacteroides in the control older age (>60 years old) subgroup was higher than that in the T2DM older age subgroup ( $\mathrm{P}=0.0208$; Fig. $3 \mathrm{~A})$. The abundance of Bifidobacterium in the T2DM older age subgroup ( $>60$ years old) was higher than that in the T2DM younger age ( $\leq 60$ years old) subgroup $(\mathrm{P}=0.0343)$ and the control older age subgroup $(\mathrm{P}=0.0041$; Fig. 3B). The abundance of Enterococcus was significantly higher in both the T2DM older age and younger age subgroups $(\mathrm{P}=0.0012$ and $\mathrm{P}=0.0012$, respectively; Fig. 3C), compared with control participants less than 60 years old. Furthermore, Roseburia inulinivorans was significantly higher in the control younger age subgroup than in the T2DM older age subgroup ( $\mathrm{P}=0.0007$; Fig. 3D). However, there were no significant differences in the abundance of Prevotella $(\mathrm{P}=0.0975)$, Lactobacillus $(\mathrm{P}=0.0697)$, Eubacterium rectale $(\mathrm{P}=0.102)$, Faecalibacterium prausnitzii $(\mathrm{P}=0.455)$, Veillonellaceae $(\mathrm{P}=0.507)$ and Clostridium leptum $(\mathrm{P}=0.904)$ between each subgroup (Fig. S3). 
A

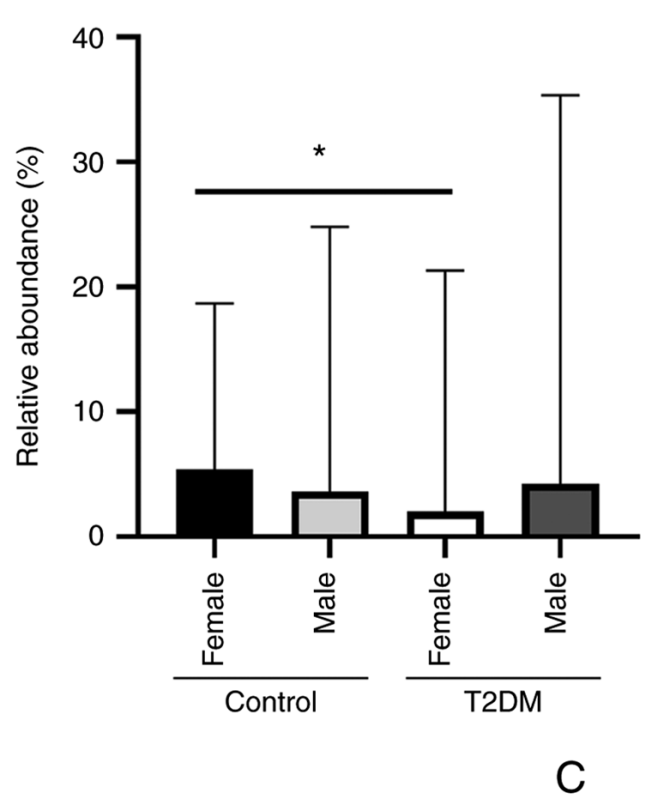

B

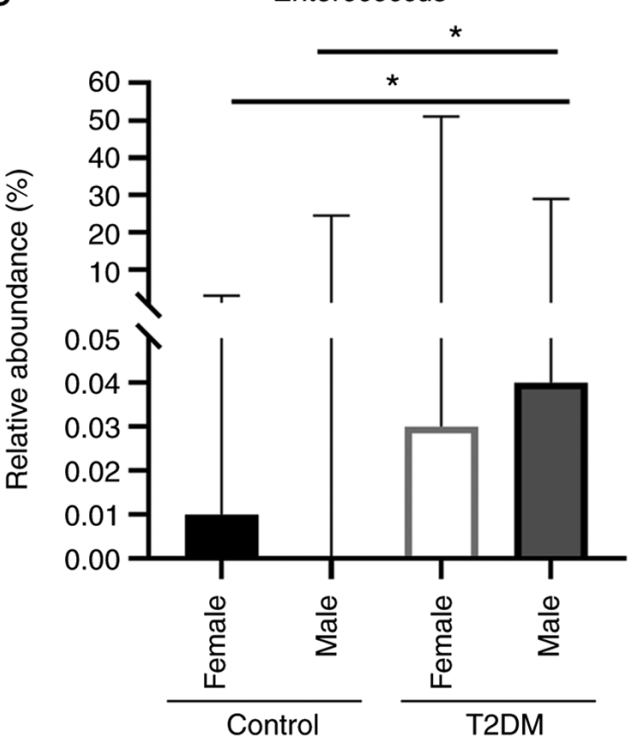

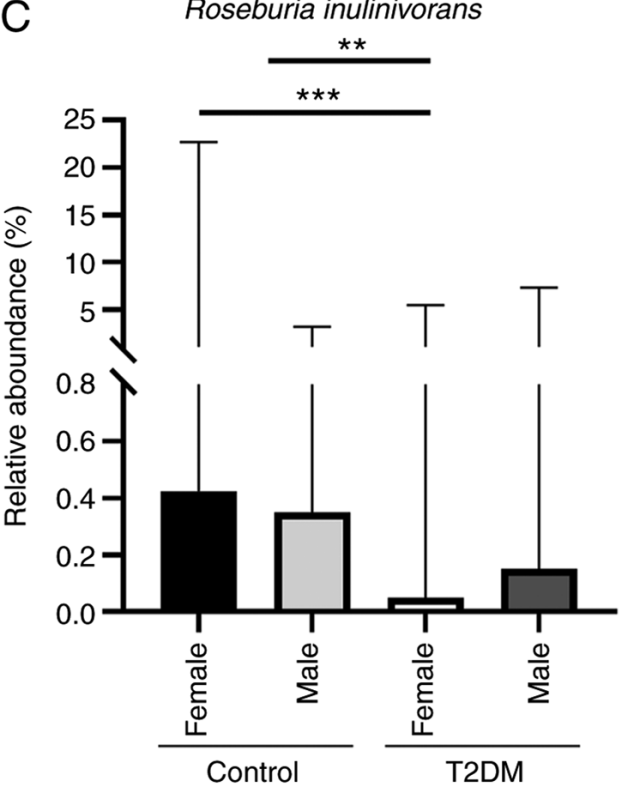

Figure 2. Comparison of 3 bacterial species between the control female and male subgroups, and the T2DM female and male subgroups. (A) Faecalibacterium prausnitzii abundance was significantly higher in the control female subgroup than in the T2DM female subgroup. (B) The abundance of Enterococcus was higher in the T2DM male subgroup than in both control female and male subgroups. (C) The abundance of Roseburia inulinivorans was significantly higher in both control female and male subgroups than in the T2DM female subgroup. Results represent the median and range. ${ }^{*} \mathrm{P}<0.05,{ }^{* * *} \mathrm{P}<0.01$ and ${ }^{* * * *} \mathrm{P}<0.001$ as determined by Kruskal-Wallis test followed by Dunn's post hoc test. T2DM, type 2 diabetes mellitus.

Comparison of the 3 machine learning models. SVM, XGboost and MLP models were used to predict the risk of T2DM by incorporating 6 clinical features and 10 bacterial species. A total of 207 samples were randomly divided into a training set $(80 \%)$ and test set $(20 \%)$. The ROC curve is widely used to validate the performance of prediction models, and the average AUC and $95 \%$ CI are shown in Fig. 4A. In the training set, the results indicated that the AUC values of SVM, XGboost and MLP models were 0.72, 0.70 and 0.69, respectively. Furthermore, the accuracy, ppv (precision) and sensitivity (recall) were $>0.61$ in all models (Table SIV). However, specificity and npv were poor in the three models. In the test set, the results showed that the SVM obtained the highest AUC value (0.77); the XGboost and MLP model
AUC values were 0.69 and 0.67 , respectively (Fig. 4B). The accuracy was $>0.67$ in the three models and the specificity and precision were $>0.72$ (Table SV). However, recall and npv did not perform well in all the models. Furthermore, the XGboost model was used to analyze the importance of the 16 features, and then the feature score rankings were measured (Fig. 5). The results showed that Bifidobacterium, age and Roseburia inulinivorans were the top three features in the model.

\section{Discussion}

The prevalence of T2DM has become a major public concern, with its continuingly increasing incidence worldwide. Gut 
A

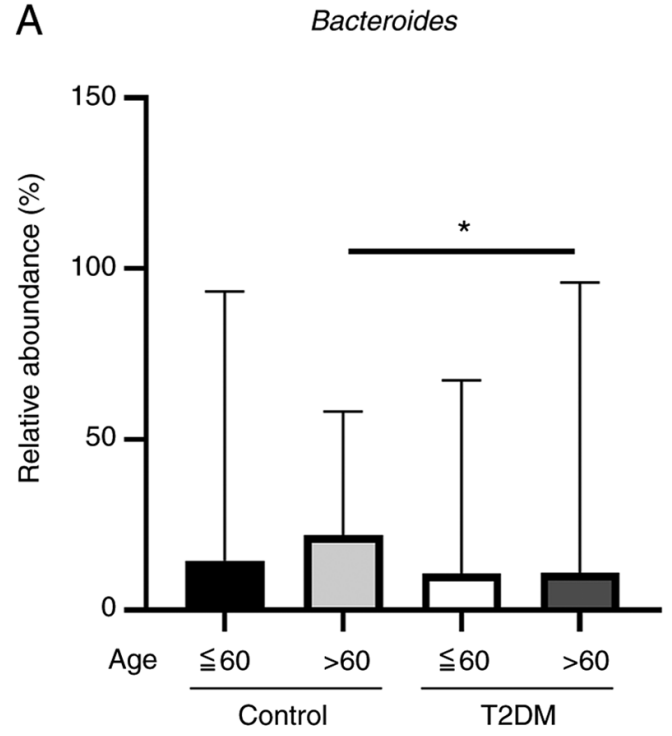

C

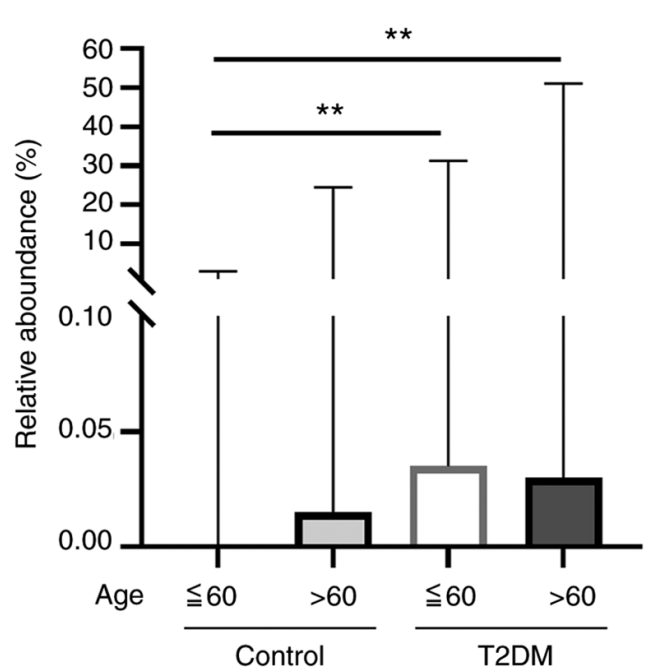

B

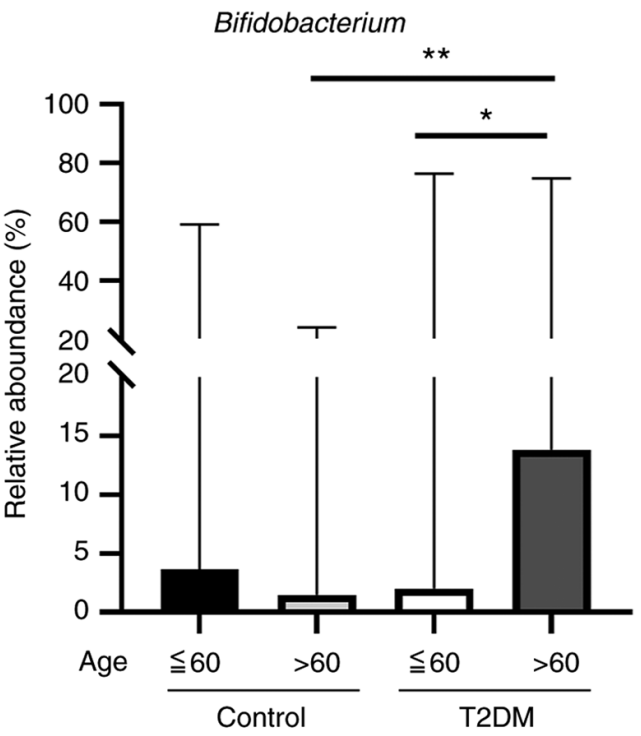

D

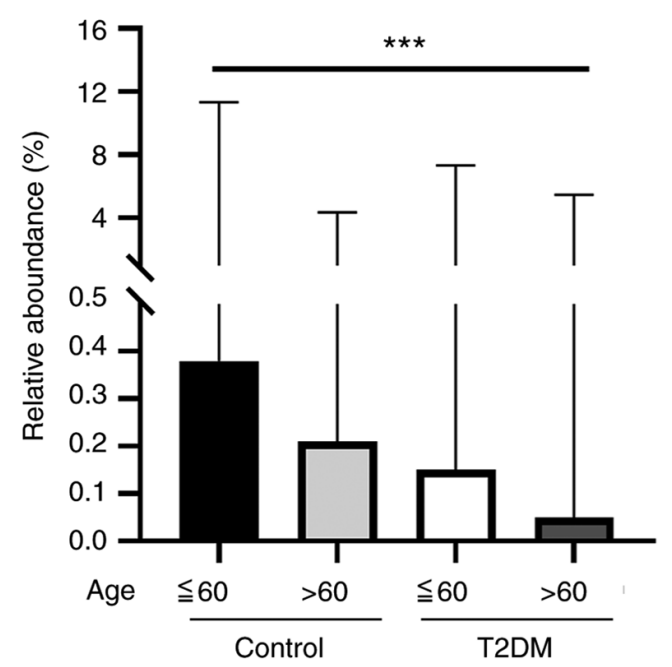

Figure 3. Comparison of 4 bacterial species between the control older age and younger age subgroups, and the T2DM older age and younger age subgroups. (A) The abundance of Bacteroides was higher in the T2DM older age subgroup than in the control older age subgroup. (B) The abundance of Bifidobacterium was higher in the T2DM older age subgroup than in the T2DM younger age subgroup and the control older age subgroup. (C) The abundance of Enterococcus was significantly higher in both the T2DM younger age and older age subgroups than in the control younger age subgroup. (D) Abundance of Roseburia inulinivorans was significantly higher in the control younger age subgroup than in the T2DM older age subgroup. Results represent the median and range. ${ }^{*} \mathrm{P}<0.05,{ }^{* *} \mathrm{P}<0.01$ and ${ }^{* * *} \mathrm{P}<0.001$ as determined by Kruskal-Wallis test followed by Dunn's post hoc test. T2DM, type 2 diabetes mellitus.

dysbiosis in patients with T2DM is caused by not only environmental factors but also the host genetics. Studies have suggested that the composition of the intestinal microbiota can trigger T2DM (25-27). Therefore, further research is required to elucidate the connection between GM and T2DM.

T2DM is a systemic disease which is characterized by hyperglycemia, hyperlipidemia and organismic insulin resistance (28). In the present study, six clinical data including age, sex, BMI, smoking, alcohol consumption and hypertension status which were associated with T2DM development were collected. Additionally, numerous studies have shown that non-alcoholic fatty liver disease (NAFLD) is commonly observed in patients with T2DM $(29,30)$. However, whether NAFLD is a cause or consequence of the diabetic pathology remains a source of debate (28). Thus, this relationship is bidirectional, since T2DM substantially predicts the development of these metabolic disorders (3). Therefore, the NAFLD status, hyperlipidemia and disorders of glucose and lipid metabolism status were not collected to predict the risk of T2DM in the present study. According to previous studies, 10 bacteria including Veillonellaceae, Clostridium leptum, Roseburia inulinivorans, Bacteroides, Prevotella, Bifidobacterium, Lactobacillus, Faecalibacterium prausnitzii, Enterococcus and Eubacterium rectale were associated with T2DM $(31,32)$. The abundance of Bacteroides was significantly lower in the T2DM group, which is consistent with a previous study in animals, which revealed that after administration of metformin, the relative abundance of Bacteroides was increased in mice and rats treated with metformin (33). Roseburia inulinivorans was more abundant in the control group in the present study, which is similar to a study which showed that the abundance of Roseburia inulinivorans was increased in patients after 
A

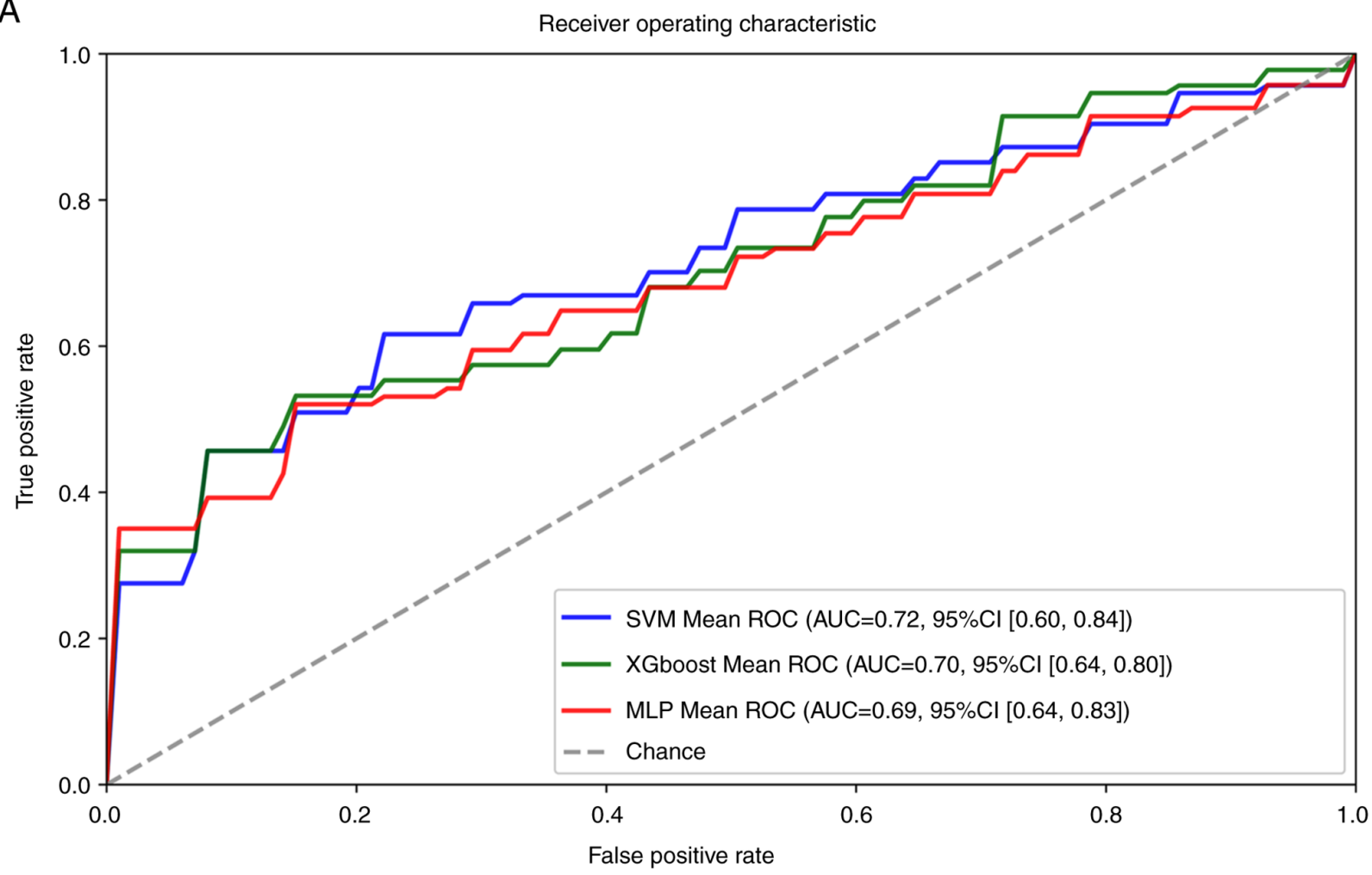

B Receiver operating characteristic

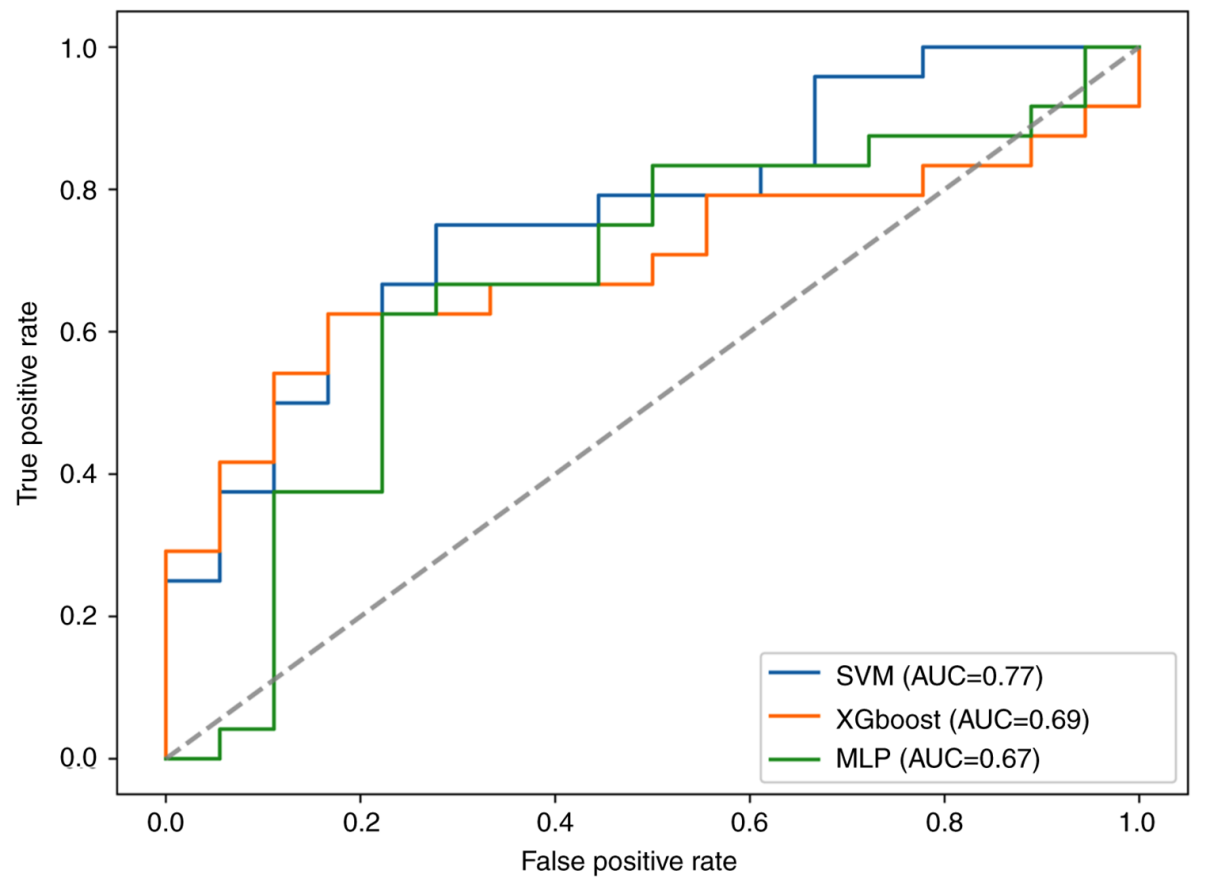

Figure 4. Evaluation of the predictive models. The figure shows the average ROC curves of the 3 models in the training set and test set. (A) Mean AUC values and 95\% CIs of all models are shown in the training set. (B) The AUC values of all models are shown in the test set. ROC, receiver operating characteristic; AUC, area under the ROC curve; CI, confidence interval.

diabetic remission, achieved by both laparoscopic Roux-en-Y gastric bypass or sleeve gastrectomy surgery (34). Interestingly, Roseburia inulinivorans was significantly higher in both control female and male subgroups than in the T2DM female subgroup. Moreover, Roseburia inulinivorans was significantly higher in the control younger age subgroup than in the T2DM older age subgroup. In the present study, the abundance of Eubacterium rectale was significantly higher in the control group than in the T2DM group, which is consistent with a metagenome-wide association study which revealed that the relative abundance of Eubacterium rectale was higher in the control group than in the patients with T2DM (31). A previous study found that 


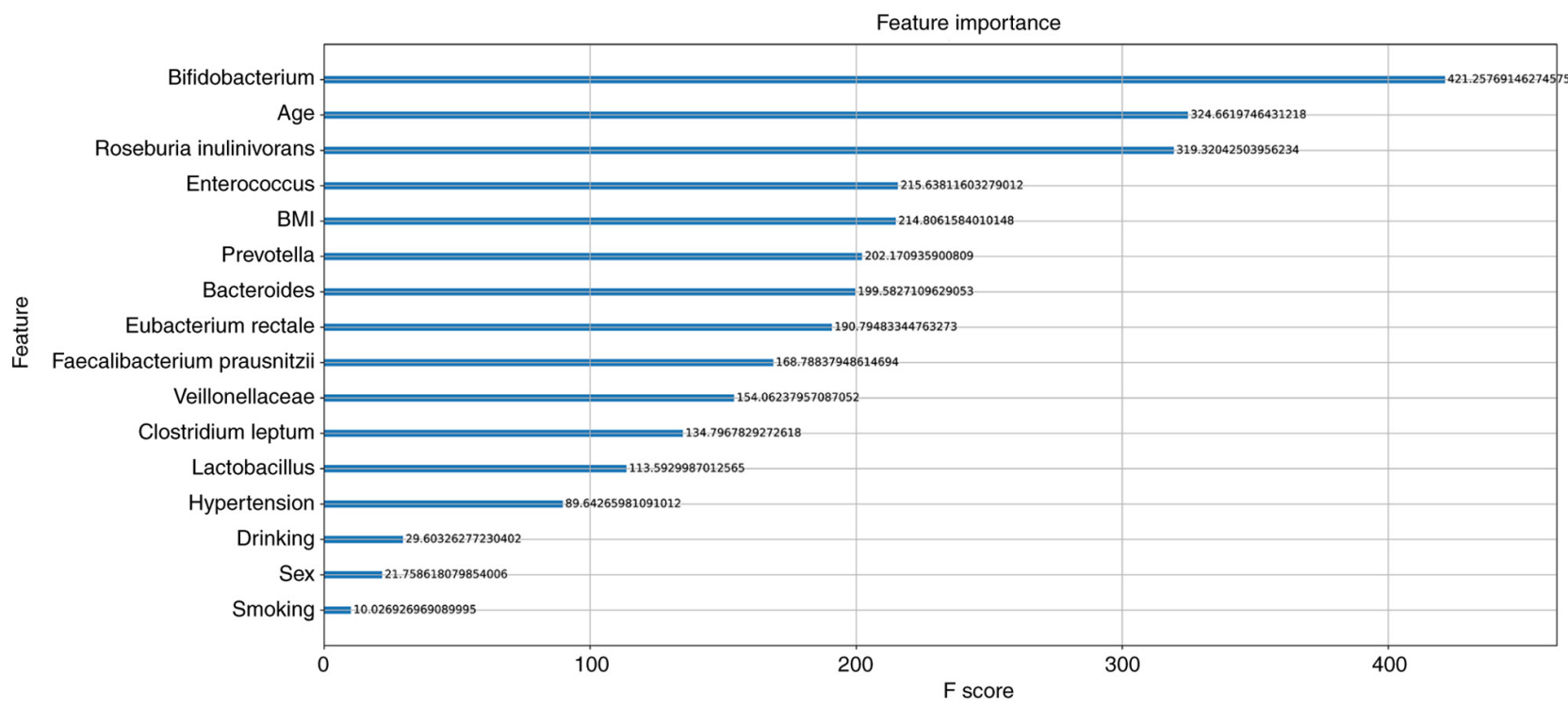

Figure 5. Evaluation of the predictive models. The figure shows the average ROC curves of the 3 models in the training set and test set. (A) Mean AUC values and $95 \%$ CIs of all models are shown in the training set. (B) The AUC values of all models are shown in the test set. ROC, receiver operating characteristic; AUC, area under the ROC curve; CI, confidence interval.

Enterococcus was positively correlated with obesity (35). Enterococcus was significantly higher in the patients with T2DM in the present study, which is in accordance with a study that showed that Enterococcus was more enriched in the DM group than in the control group (36). Interestingly, the abundance of Enterococcus was higher in both T2DM younger and older age subgroups than in the control younger age subgroup. Additionally, Enterococcus was higher in the T2DM male subgroup than in both control female and male subgroups.

Additionally, several studies have shown that Bifidobacterium was negatively associated with T2DM $(37,38)$. Conversely, Sasaki et al (39) reported that Bifidobacterium was significantly increased in the patients with T2DM when compared with the healthy controls. However, in the present study, the abundance of Bifidobacterium did not significantly differ between the control group and the T2DM group. Interestingly, Bifidobacterium exhibited the higher abundance in the T2DM older age subgroup than in the T2DM younger age subgroup and the control older age subgroup. Faecalibacterium prausnitzii was found to be negatively associated with T2DM $(32,40)$. Interestingly, Faecalibacterium prausnitzii was significantly higher in the control female subgroup than in the T2DM female subgroup; although there was no significant difference in Faecalibacterium prausnitzii abundance between the control group and T2DM group. Penckofer et al (41) reported that Lactobacillus was more abundant in women with T2DM than in the controls. Conversely, previous studies have demonstrated the beneficial effects of Lactobacillus for human health, including improving T2DM, exhibiting anti-inflammatory effects and reducing body weight (42-44). Human gut Lactobacillus can reduce blood glucose responses in vivo (45). The aforementioned studies indicated that Lactobacillus shows the most discrepant results among studies. Furthermore, Prevotella was significantly correlated with lipid metabolites, such as lysophosphatidylglycerol and phosphatidylinositol-3, resembling obese and diabetic phenotypes (46). The abundance of
Clostridium leptum in the probiotic group was significantly higher than in the control group who did not take probiotics than in Japanese patients with T2DM (47). In a previous study, it was demonstrated that Veillonellaceae was significantly higher in the acarbose group than in the placebo group (48). However, in the present study, the abundance of Lactobacillus, Prevotella, Clostridium leptum and Veillonellaceae did not significantly differ between the control and T2DM groups.

In order to improve earlier warnings in patients with T2DM, the SVM, XGboost and MLP models were used, incorporating 6 clinical features and 10 bacterial species to predict the risk of T2DM. A total of 207 samples were randomly divided into a training set $(80 \%)$ and test set $(20 \%)$. Among the three models, SVM and XGboost models obtained AUC values of 0.72 and 0.70 , respectively, in the training set, and the accuracy, precision and recall were $>0.61$. While in the test set, only the SVM model obtained an AUC value of 0.77, the precision and specificity were $>0.77$, and the accuracy, recall, and npv were $>0.60$. Previous studies reported that if the model AUC is $>0.70$, the model has high accuracy $(49,50)$. Although the SVM model had the highest overall predictive power, the sample size in the training and test set were small. Thus, large samples are required to verify this result.

In addition, the XGboost model was used to analyze the importance of the 16 features, including 6 clinical features and 10 bacterial species. The results revealed that Bifidobacterium, age and Roseburia inulinivorans played major roles in the model, while alcohol consumption, smoking status and sex were less important. Bifidobacterium represents beneficial genera, most frequently reported in studies of T2DM, and appears to be the most consistent genus supported by the literature, exhibiting potentially protective effects against T2DM (51). Roseburia inulinivorans is also the most consistently reported to exhibit a negative association with T2DM (51). Therefore, the results indicated that the gut microbiome can be a potential marker for predicting the risk of T2DM. Yang et al (52) reported that the meta-analysis of 
the prevalence T2DM rate at the age of 55-74 years was six- to seven-fold higher than that of individuals aged 20-34 years, in China. Thus, age may be a major factor in the risk of T2DM. In the present study, age was ranked as the second most important factor in the model. Additionally, Bifidobacterium, Roseburia inulinivorans and Enterococcus were associated with an older age. Bifidobacterium, Roseburia inulinivorans and Enterococcus were ranked as the top 5 important features in the model. In addition, a previous study showed that a high BMI was the single strongest risk factor for T2DM (53), and was associated with several metabolic abnormalities that result in insulin resistance (54). According to a series of nationwide surveys reported in China (55), the prevalence of being overweight $\left(23 \mathrm{~kg} / \mathrm{m}^{2} \leq \mathrm{BMI}<27.5 \mathrm{~kg} / \mathrm{m}^{2}\right)$ in Chinese adults aged 20-59 years old increased from $37.4 \%$ in 2000 to $39.2 \%$ in $2005,40.7 \%$ in 2010 , and $41.2 \%$ in 2014 . The prevalence of obesity (BMI $\geq 27.5 \mathrm{~kg} / \mathrm{m}^{2}$ ) increased from $8.6 \%$ in 2000 to $10.3 \%$ in $2005,12.2 \%$ in 2010 and $12.9 \%$ in 2014 . Notably, T2DM develops at a considerably lower BMI in the Chinese population than in European populations. The relatively high risk of diabetes at a lower BMI could be partially attributed to the tendency towards visceral adiposity in East Asian populations, including the Chinese population (56). Therefore, BMI may not play an important role in developing T2DM in the Chinese population. In the present study, BMI ranked as the fifth most important feature in the model. In addition, smoking has shown to induce insulin resistance and compensatory insulin-secretion responses (57), which may explain the increased risk of T2DM in individuals who smoke. On the one hand, moderate consumption of alcohol has been associated with a reduced risk of T2DM (8). On the other hand, it may be due to the public education campaigns to reduce the prevalence of smoking in China in recent years. A meta-analysis indicated that the prevalence of T2DM was 9.9\% for men and $11.6 \%$ for women in China (2000-2014) (52). It appears that the effect of sex on the prevalence of T2DM amongst the Chinese is equal. Thus, alcohol consumption, smoking and sex are less important in the model ranking. Meanwhile, the abundance of Faecalibacterium prausnitzii, Veillonellaceae, Clostridium leptum and Lactobacillus did not differ between the control and T2DM groups, which may explain why they were ranked lower.

There are several limitations in the present study. First, the sample size used was relatively small and the total cohort of patients with T2DM and cohort of controls was unbalanced. Second, only 6 clinical features and 10 bacterial species were used to establish the models between the two groups. The $16 \mathrm{~S}$ rRNA gene is a promising method for detecting GM, but in the present study, the abundance of the 10 bacterial species between the two groups was assessed by qPCR instead. Third, although the SVM model obtained an AUC value of 0.77 in the test set, larger cohorts are required to validate in the model before the model can be assessed in the clinic for detection of early stage T2DM.

In conclusion, three machine learning models were constructed and compared to predict the risk of T2DM, revealing that the SVM model exhibited the highest overall predictive power. In addition, Bifidobacterium, age and Roseburia inulinivorans had important impacts in predicting early stage T2DM. Therefore, SVM machine learning may have potential to aid in the early prediction and treatment of patients with T2DM in the near feature.

\section{Acknowledgements}

Not applicable.

\section{Funding}

No funding was received.

\section{Availability of data and materials}

The datasets used and/or analyzed during the present study are available from the corresponding author on reasonable request.

\section{Authors' contributions}

XG, HT, JZ and YG designed the experiments. AZ, LL, QS, $\mathrm{JH}$ and $\mathrm{YW}$ collected the samples and performed the experiments. XG, RT, YX, JZ and YP analyzed the data. XG, HT, JZ and YG confirm the authenticity of all the raw data. XG, HT, YX and YG wrote the manuscript. JZ, YX, HT and YG revised the manuscript. All authors have read and approved the final manuscript.

\section{Ethics approval and informed consent}

The present study was approved (approval no. CYFYLL2021171) by the Ethics Committee of the affiliated Hospital of Chengde Medical University (Chengde, China). Written informed consent was obtained from all participants involved in the present study.

\section{Patient consent for publication}

Not applicable.

\section{Competing interests}

The authors declare that they have no competing interests.

\section{References}

1. Roden M and Shulman GI: The integrative biology of type 2 diabetes. Nature 576: 51-60, 2019.

2. Massey W and Brown JM: The gut microbial endocrine organ in type 2 diabetes. Endocrinology 162: bqaa235, 2021.

3. Hu C and Jia W: Diabetes in China: Epidemiology and genetic risk factors and their clinical utility in personalized medication. Diabetes 67: 3-11, 2018.

4. Pavlou DI, Paschou SA, Anagnostis P, Spartalis M, Spartalis E, Vryonidou A, Tentolouris N and Siasos G: Hypertension in patients with type 2 diabetes mellitus: Targets and management. Maturitas 112: 71-77, 2018.

5. Colosia AD, Palencia R and Khan S: Prevalence of hypertension and obesity in patients with type 2 diabetes mellitus in observational studies: A systematic literature review. Diabetes Metab Syndr Obes 6: 327-338, 2013.

6. Sabuncu T, Sonmez A, Eren MA, Sahin I, Çorapçioğlu D, Üçler R, Akin S, Haymana C, Demirci İ, Atmaca A, et al: Characteristics of patients with hypertension in a population with type 2 diabetes mellitus. Results from the Turkish Nationwide SurvEy of Glycemic and other metabolic parameters of patients with diabetes mellitus (TEMD Hypertension Study). Prim Care Diabetes 15: 332-339, 2021 
7. National high blood pressure education program working group report on hypertension in diabetes. Hypertension 23: 145-158; discussion 159-160, 1994.

8. Baliunas DO, Taylor BJ, Irving H, Roerecke M, Patra J, Mohapatra S and Rehm J: Alcohol as a risk factor for type 2 diabetes: A systematic review and meta-analysis. Diabetes Care 32: 2123-2132, 2009.

9. Ezzati $\mathrm{M}$ and Riboli E: Behavioral and dietary risk factors for noncommunicable diseases. N Engl J Med 369: 954-964, 2013.

10. Powles JW, Zatonski W, Vander Hoorn S and Ezzati M: The contribution of leading diseases and risk factors to excess losses of healthy life in Eastern Europe: Burden of disease study. BMC Public Health 5: 116, 2005.

11. Zhou H, Sun L, Zhang S, Zhao X, Gang X and Wang G: Evaluating the causal role of gut microbiota in type 1 diabetes and its possible pathogenic mechanisms. Front Endocrinol (Lausanne) 11: 125, 2020

12. Hasain Z, Mokhtar NM, Kamaruddin NA, Mohamed Ismail NA, Razalli NH, Gnanou JV and Raja Ali RA: Gut microbiota and gestational diabetes mellitus: A review of host-gut microbiota interactions and their therapeutic potential. Front Cell Infect Microbiol 10: 188, 2020.

13. Gilbert JA, Blaser MJ, Caporaso JG, Jansson JK, Lynch SV and Knight R: Current understanding of the human microbiome. Nat Med 24: 392-400, 2018

14. Takagi $T$, Naito $Y$, Inoue $R$, Kashiwagi S, Uchiyama $K$, Mizushima K, Tsuchiya S, Dohi O, Yoshida N, Kamada K, et al: Differences in gut microbiota associated with age, sex, and stool consistency in healthy Japanese subjects. J Gastroenterol 54: 53-63, 2019.

15. Pasolli E, Asnicar F, Manara S, Zolfo M, Karcher N, Armanini F, Beghini F, Manghi P, Tett A, Ghensi P, et al: Extensive unexplored human microbiome diversity revealed by over 150,000 genomes from metagenomes spanning age, geography, and lifestyle. Cell 176: 649-662.e620, 2019.

16. Alberti KG and Zimmet PZ: Definition, diagnosis and classification of diabetes mellitus and its complications. Part 1: Diagnosis and classification of diabetes mellitus provisional report of a WHO consultation. Diabet Med 15: 539-553, 1998.

17. Lin Q, Zhou W, Wang Y, Huang J, Hui X, Zhou Z and Xiao Y: Abnormal peripheral neutrophil transcriptome in newly diagnosed type 2 diabetes patients. J Diabetes Res 2020: 9519072 , 2020.

18. Livak KJ and Schmittgen TD: Analysis of relative gene expression data using real-time quantitative PCR and the 2(-Delta Delta C(T)) method. Methods 25: 402-408, 2001.

19. Xiao J, Ding R, Xu X, Guan H, Feng X, Sun T, Zhu S and Ye Z: Comparison and development of machine learning tools in the prediction of chronic kidney disease progression. J Transl Med 17: 119, 2019

20. Yao RQ, Jin X, Wang GW, Yu Y, Wu GS, Zhu YB, Li L, Li YX, Zhao PY, Zhu SY, et al: A machine learning-based prediction of hospital mortality in patients with postoperative sepsis. Front Med (Lausanne) 7: 445, 2020.

21. Souza Filho JB, Sanchez M, Seixas JM, Maidantchik C, Galliez R, Moreira AD, da Costa PA, Oliveira MM, Harries AD and Kritski AL: Screening for active pulmonary tuberculosis: Development and applicability of artificial neural network models. Tuberculosis (Edinb) 111: 94-101, 2018.

22. Vabalas A, Gowen E, Poliakoff E and Casson AJ: Machine learning algorithm validation with a limited sample size. PLoS One 14: e0224365, 2019.

23. Ma X, Wu Y, Zhang L, Yuan W, Yan L, Fan S, Lian Y, Zhu X, Gao J, Zhao J, et al: Comparison and development of machine learning tools for the prediction of chronic obstructive pulmonary disease in the Chinese population. J Transl Med 18: 146, 2020.

24. Wu D, Yang Q, Su B, Hao J, Ma H, Yuan W, Gao J, Ding F, $\mathrm{Xu} \mathrm{Y}$, Wang H, et al: Low-density lipoprotein cholesterol 4: The notable risk factor of coronary artery disease development. Front Cardiovasc Med 8: 619386, 2021.

25. Sircana A, Framarin L, Leone N, Berrutti M, Castellino F, Parente R, De Michieli F, Paschetta E and Musso G: Altered gut microbiota in type 2 diabetes: Just a coincidence? Curr Diab Rep 18: 98, 2018.

26. Salgaco MK, Oliveira LG, Costa GN, Bianchi F and Sivieri K: Relationship between gut microbiota, probiotics, and type 2 diabetes mellitus. Appl Microbiol Biotechnol 103: 9229-9238, 2019.
27. Wu Q, Wu S, Cheng Y, Zhang Z, Mao G, Li S, Yang Y, Zhang X, $\mathrm{Wu} \mathrm{M}$ and Tong $\mathrm{H}$ : Sargassum fusiforme fucoidan modifies gut microbiota and intestinal metabolites during alleviation of hyperglycemia in type 2 diabetic mice. Food Funct 12: 3572-3585, 2021.

28. Pinti MV, Fink GK, Hathaway QA, Durr AJ, Kunovac A and Hollander JM: Mitochondrial dysfunction in type 2 diabetes mellitus: An organ-based analysis. Am J Physiol Endocrinol Metab 316: E268-E285, 2019

29. Masuoka $\mathrm{HC}$ and Chalasani N: Nonalcoholic fatty liver disease: An emerging threat to obese and diabetic individuals. Ann N Y Acad Sci 1281: 106-122, 2013.

30. Targher G and Byrne CD: Clinical review: Nonalcoholic fatty liver disease: A novel cardiometabolic risk factor for type 2 diabetes and its complications. J Clin Endocrinol Metab 98: 483-495, 2013

31. Qin J, Li Y, Cai Z, Li S, Zhu J, Zhang F, Liang S, Zhang W, Guan Y, Shen D, et al: A metagenome-wide association study of gut microbiota in type 2 diabetes. Nature 490: 55-60, 2012.

32. Karlsson FH, Tremaroli V, Nookaew I, Bergström G, Behre CJ, Fagerberg B, Nielsen $\mathrm{J}$ and Bäckhed F: Gut metagenome in European women with normal, impaired and diabetic glucose control. Nature 498: 99-103, 2013.

33. Zhang Q and Hu N: Effects of metformin on the gut microbiota in obesity and type 2 diabetes mellitus. Diabetes Metab Syndr Obes 13: 5003-5014, 2020.

34. Murphy R, Tsai P, Jüllig M, Liu A, Plank L and Booth M: Differential changes in gut microbiota after gastric bypass and sleeve gastrectomy bariatric surgery vary according to diabetes remission. Obes Surg 27: 917-925, 2017.

35. Qiao Y, Sun J, Ding Y, Le G and Shi Y: Alterations of the gut microbiota in high-fat diet mice is strongly linked to oxidative stress. Appl Microbiol Biotechnol 97: 1689-1697, 2013.

36. Zhao X, Zhang Y, Guo R, Yu W, Zhang F, Wu F and Shang J: The alteration in composition and function of gut microbiome in patients with type 2 diabetes. J Diabetes Res 2020: 8842651, 2020.

37. Gao R, Zhu C, Li H, Yin M, Pan C, Huang L, Kong C, Wang X, Zhang Y, Qu S and Qin H: Dysbiosis signatures of gut microbiota along the sequence from healthy, young patients to those with overweight and obesity. Obesity (Silver Spring) 26: 351-361, 2018.

38. Sedighi M, Razavi S, Navab-Moghadam F, Khamseh ME, Alaei-ShahmiriF, Mehrtash A and Amirmozafari N: Comparison of gut microbiota in adult patients with type 2 diabetes and healthy individuals. Microb Pathog 111: 362-369, 2017.

39. Sasaki M, Ogasawara N, Funaki Y, Mizuno M, Iida A, Goto C Koikeda S, Kasugai K and Joh T: Transglucosidase improves the gut microbiota profile of type 2 diabetes mellitus patients: A randomized double-blind, placebo-controlled study. BMC Gastroenterol 13: 81, 2013.

40. Zhang X, Shen D, Fang Z, Jie Z, Qiu X, Zhang C, Chen Y and Ji L: Human gut microbiota changes reveal the progression of glucose intolerance. PLoS One 8: e71108, 2013.

41. Penckofer S, Limeira R, Joyce C, Grzesiak M, Thomas-White K and Wolfe AJ: Characteristics of the microbiota in the urine of women with type 2 diabetes. J Diabetes Complications 34: 107561,2020

42. Yadav H, Jain S and Sinha PR: Antidiabetic effect of probiotic dahi containing Lactobacillus acidophilus and Lactobacillus casei in high fructose fed rats. Nutrition 23: 62-68, 2007.

43. Naito E, Yoshida Y, Makino K, Kounoshi Y, Kunihiro S, Takahashi R, Matsuzaki T, Miyazaki K and Ishikawa F: Beneficial effect of oral administration of Lactobacillus casei strain Shirota on insulin resistance in diet-induced obesity mice. J Appl Microbiol 110: 650-657, 2011.

44. Kang JH, Yun SI and Park HO: Effects of Lactobacillus gasseri BNR17 on body weight and adipose tissue mass in diet-induced overweight rats. J Microbiol 48: 712-714, 2010.

45. Panwar H, Calderwood D, Grant IR, Grover S and Green BD: Lactobacillus strains isolated from infant faeces possess potent inhibitory activity against intestinal alpha- and beta-glucosidases suggesting anti-diabetic potential. Eur J Nutr 53: 1465-1474, 2014.

46. Liu H, Pan LL, Lv S, Yang Q, Zhang H, Chen W, Lv Z and Sun J: Alterations of gut microbiota and blood lipidome in gestational diabetes mellitus with hyperlipidemia. Front Physiol 10: 1015, 2019.

47. Sato J, Kanazawa A, Azuma K, Ikeda F, Goto H, Komiya K, Kanno R, Tamura Y, Asahara T, Takahashi T, et al: Probiotic reduces bacterial translocation in type 2 diabetes mellitus: A randomised controlled study. Sci Rep 7: 12115, 2017. 
48. Zhang X, Fang Z, Zhang C, Xia H, Jie Z, Han X, Chen Y and Ji L: Effects of acarbose on the gut microbiota of prediabetic patients: A randomized, double-blind, controlled crossover trial. Diabetes Ther 8: 293-307, 2017.

49. Luo X, Lin F, Zhu S, Yu M, Zhang Z, Meng L and Peng J: Mine landslide susceptibility assessment using IVM, ANN and SVM models considering the contribution of affecting factors. PLoS One 14: e0215134, 2019.

50. Hao S, Bai J, Liu H, Wang L, Liu T, Lin C, Luo X, Gao J, Zhao J, $\mathrm{Li} \mathrm{H}$ and Tang $\mathrm{H}$ : Comparison of machine learning tools for the prediction of AMD based on genetic, age, and diabetes-related variables in the Chinese population. Regen Ther 15: 180-186, 2020

51. Gurung M, Li Z, You H, Rodrigues R, Jump DB, Morgun A and Shulzhenko N: Role of gut microbiota in type 2 diabetes pathophysiology. EBioMedicine 51: 102590, 2020.

52. Yang L, Shao J, Bian Y, Wu H, Shi L, Zeng L, Li W and Dong J: Prevalence of type 2 diabetes mellitus among inland residents in China (2000-2014): A meta-analysis. J Diabetes Investig 7: 845-852, 2016.

53. Hu FB, Manson JE, Stampfer MJ, Colditz G, Liu S, Solomon CG and Willett WC: Diet, lifestyle, and the risk of type 2 diabetes mellitus in women. N Engl J Med 345: 790-797, 2001.

54. Sinha R, Dufour S, Petersen KF, LeBon V, Enoksson S, Ma YZ, Savoye M, Rothman DL, Shulman GI and Caprio S: Assessment of skeletal muscle triglyceride content by (1) $\mathrm{H}$ nuclear magnetic resonance spectroscopy in lean and obese adolescents: Relationships to insulin sensitivity, total body fat, and central adiposity. Diabetes 51: 1022-1027, 2002.
55. Tian Y, Jiang C, Wang M, Cai R, Zhang Y, He Z, Wang H, Wu D, Wang F, Liu X, et al: BMI, leisure-time physical activity, and physical fitness in adults in China: Results from a series of national surveys, 2000-14. Lancet Diabetes Endocrinol 4: 487-497, 2016.

56. Nazare JA, Smith JD, Borel AL, Haffner SM, Balkau B, Ross R, Massien C, Alméras N and Després JP: Ethnic influences on the relations between abdominal subcutaneous and visceral adiposity, liver fat, and cardiometabolic risk profile: The international study of prediction of intra-abdominal adiposity and its relationship with cardiometabolic risk/intra-abdominal adiposity. Am J Clin Nutr 96: 714-726, 2012.

57. Reaven G and Tsao PS: Insulin resistance and compensatory hyperinsulinemia: The key player between cigarette smoking and cardiovascular disease? J Am Coll Cardiol 41: 1044-1047, 2003.

This work is licensed under a Creative Commons Attribution-NonCommercial-NoDerivatives 4.0 International (CC BY-NC-ND 4.0) License. 